\title{
New chart to evaluate weight faltering
}

\author{
Charlotte Wright, Alison Avery, Melanie Epstein, Eileen Birks, Dominic Croft
}

\begin{abstract}
A new chart was designed to aid accurate identification of weight faltering and failure to thrive. It provides guidance on the lower limits of expected weight gain for children, whatever their initial centile position. The chart's theoretical basis, the process of its construction, and its evaluation are described in this paper.

Evaluation was by a self completion questionnaire, where respondents answered questions about a range of standardised growth patterns, plotted on old and new charts. Forty five health visitors, 28 general practitioner principals and registrars, and nine community paediatricians provided 328 chart ratings. These showed that the new format significantly increased the proportion of correctly rated charts (old: 45 (28\%); new: 82 $(51 \%))$, with the greatest impact in severe cases. This suggests that the new chart improves the precision of judgments made about weight gain in infancy.

(Arch Dis Child 1998;78:40-43)
\end{abstract}

Keywords: weight monitoring; failure to thrive; regression to the mean

Health practitioners are accustomed to using conventional cross sectional charts for tracking weight gain over time, although such charts actually offer no explicit guidance on when a fall down a centile chart becomes a cause for concern.

Recent publications have highlighted this deficiency in cross sectional charts and advocated the development of conditional weight charts for this purpose. ${ }^{12}$ An impediment to evaluating falls down a centile chart is the phenomenon of regression to the mean, that is, where large babies tend to show falls towards average while small babies may move upwards. This means that consistent guidance about what constitutes a normal fall down a standard cross sectional chart cannot easily be offered. The solution to this problem in our research practice has been to develop the thrive index methodology, ${ }^{3}$ which we have now been using for five years to screen and monitor children with failure to thrive. ${ }^{4}$ This uses a weight or weights in the early weeks as a baseline for comparison with subsequent weights after adjustment for regression to the mean. This methodology, however, requires the transformation of weights into SD scores using a computer program and can be difficult for health professionals, let alone parents, to grasp. We recognised that a chart based interpretation of this approach is needed for the routine diagnosis and monitoring of slow weight gain in early childhood, particularly in primary care. We have developed such a chart, which we have used in our clinical service since 1995. This paper explains the theoretical principles underpinning the chart and the process of its design and describes a formal evaluation of its effectiveness.

\section{Part 1: designing the chart}

Our design was directly inspired by Carpenter's chart for monitoring children at risk of sudden infant death syndrome. ${ }^{5}$ This consists of a number of equidistant lines, closer together than those on the conventional centile chart, delineating a series of channels. Children who cross through more than a defined number of channels over two to eight weeks are identified as needing extra monitoring. This chart, however, makes no allowance for regression to the mean, which is particularly necessary when monitoring centile shifts over longer periods of time.

We therefore designed a chart with nonequidistant channels: wide at the top of the normal range and progressively narrower lower down, reflecting the tendency for large babies to fall and for smaller babies to rise in centile terms. Children's channel position in the early weeks of life can then be used to define their baseline. The number of channel widths crossed downwards from that position represents an equivalent distance of fall for children at different starting positions, and consistently determines whether a change in channel position is abnormal.

\section{DEFINING THE NORMAL LIMITS FOR CENTILE}

SHIFTS

In order to construct a chart to meet these criteria, we first had to define the relation between early and late centile position for normal children using the UK 1990 standards. As with our previous work, this comparison was made using our longitudinal weight data set from Newcastle children. ${ }^{3}$ These were weights collected routinely between birth and 24 months from a cohort of 3418 full term infants. The weights were transformed into SD scores, using the 1990 standards, corrected in $1996 .^{67} \mathrm{~A}$ baseline was calculated for each child by taking an average of all the weight SD scores available between birth and 2 months (one to three values). This baseline (SD score ${ }_{1}$ ) was then compared with the latest weight SD score available per child in the age group 9-18 months (SD score $_{2}$ ). The two values were then related using linear regression which resulted in these estimates (standard error): SD score $_{2}=\mathrm{SD}$ score $_{1} \times 0.58(0.018)+0.08(0.018)$.

This equation, rounded for simplicity, was then used to make the correction for regression to the mean and produce a prediction of 
Table 1 Actual 5\% and 1\% limits compared with those defined by chart

\begin{tabular}{|c|c|c|c|c|c|}
\hline \multicolumn{2}{|c|}{ From initial chart position } & \multicolumn{2}{|c|}{ Fall to $5 \%$ threshold ${ }^{\star}$} & \multicolumn{2}{|c|}{ Fall to $1 \%$ threshold $t$} \\
\hline $\begin{array}{l}\text { Upper limit of } \\
\text { channel }\end{array}$ & $\begin{array}{l}\text { Actual } \\
\text { starting SD } \\
\text { score }\end{array}$ & $\begin{array}{l}\text { Limit on chart } \\
\text { (SD score) }\end{array}$ & $\begin{array}{l}\text { True limit } \\
\text { (SD score) }\end{array}$ & $\begin{array}{l}\text { Limit on chart } \\
\text { (SD score) }\end{array}$ & $\begin{array}{l}\text { True limit } \\
\text { (SD score) }\end{array}$ \\
\hline 10 & 2.2 & 0 & -0.08 & -0.67 & -0.61 \\
\hline 9 & 1.1 & -0.67 & -0.74 & -1.33 & -1.27 \\
\hline 8 (50th) & 0 & -1.33 & -1.4 & -2.15 & -1.93 \\
\hline 7 (25th) & -0.67 & -1.72 & -1.80 & -2.4 & -2.34 \\
\hline 6 (9th) & -1.33 & -2.15 & -2.20 & -2.67 & -2.73 \\
\hline 5 & -1.72 & -2.4 & -2.43 & -2.85 & -2.96 \\
\hline 4 & -2.15 & -2.67 & -2.69 & -3 & -3.22 \\
\hline 3 & -2.4 & -2.85 & -2.84 & & \\
\hline 2 (0.4th) & -2.67 & -3 & -2.99 & & \\
\hline
\end{tabular}

* Through 2 channel widths.

† Through 3 channel widths from $>50$ th centile, or four channels $<50$ th.

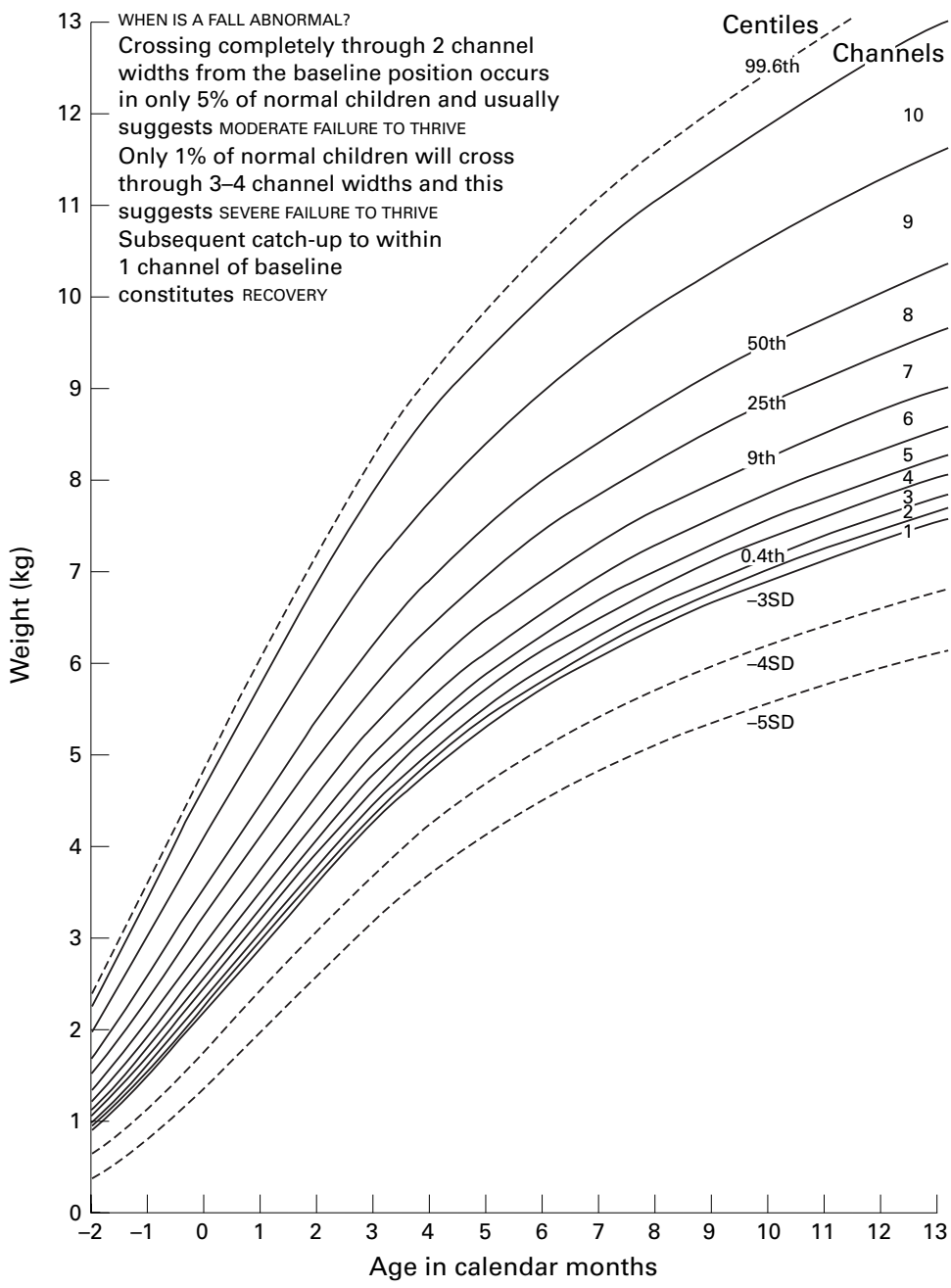

Figure 1 Schematic representation of chart for first year in boys (ages 2-5 not illustrated).

expected weight SD scores for children of 9-18 months old: predicted SD score $_{2}=$ SD score $_{1}$ $\times 0.6$.

This value was then used to calculate the difference between the actual and predicted score for every child in the cohort, which was termed the thrive index (that is, thrive index = actual SD score $_{2}$ - predicted SD score $_{2}$ ).

The normal range and lower limits of the thrive index were then calculated and this suggested that $95 \%$ of children had values above -1.4 SD score and $99 \%$ above -1.9 SD score. Using this formula, we calculated the expected lower limit of fall for children starting from a
Table 2 Permutations of growth patterns presented

\begin{tabular}{llll}
\hline Chart number & Chart format & Size of fall $^{*}$ & Starting centile \\
\hline 1 & Standard & Normal & 98 th \\
7 & New & & \\
2 & Standard & Moderate & 98 th \\
8 & New & & \\
3 & Standard & Severe & 98 th \\
9 & New & & \\
4 & Standard & Normal & 2nd \\
10 & New & & \\
5 & Standard & Moderate & 2nd \\
11 & New & & \\
6 & Standard & Severe & 2nd \\
12 & New & & \\
\hline
\end{tabular}

$\star$ Normal $=>10 \%$ threshold, fall of 1.5 channel widths. Moderate $=<5 \%$ threshold, fall of $>2.5$ channel widths. Severe $=<1 \%$ threshold, fall of 4.5 channel widths

range of initial centile positions. We have previously described similar results using the weight at 6 weeks as a baseline and comparing our dataset with the Cambridge growth standard, on which the UK 1990 standards are largely based. ${ }^{3}$ We have chosen to use an average baseline rather than the weight at 6 weeks alone, because an average of this kind is less prone to distortion by individual measurement error or short term illness.

CONSTRUCTION OF THE CHART

Using this information, we then chose a range of centile lines that met the requirement that a fall through the equivalent of two adjoining channel widths from any starting position within the normal range would constitute a fall, placing the child in the slowest gaining 5\% (a fall of 1.4 SD score). That is, a large child crossing through two wide channels at the top of the chart falls equivalently to an initially small child falling through two narrower channels at the bottom of the chart. A practical constraint on chart design is that it should be modelled as closely as possible on existing cross sectional charts, to lessen confusion when used alongside them. Thus priority was given to using the centile lines in the current nine centile chart. ${ }^{8}$ The final chart consisted of 11 lines delineating 10 channels numbered upwards (fig 1). The SD score values and centiles used, where they corresponded with standard centiles, were: $2.2,1.1,0$ (50th), -0.67 (25th), -1.33 (9th), $-1.8,-2.15,-2.4,-2.67$ (0.4th), $-2.85,-3$. Two channel widths closely represented the actual 5\% limit throughout the normal range, but it was not possible to achieve this while simultaneously producing a chart that consistently represented more severe falls through the normal range (table 1). We can state, however, that starting from above the 50th centile, a fall through three channel widths would place a child in the slowest gaining $1 \%$ of children, as would a fall through four channel widths starting from below the 50th centile. A detailed explanations of the use and interpretation of the chart is provided on its front cover, with a summary embedded in the chart.

In addition to the specified channels, guidance lines at -4 and -5 SD scores are shown for monitoring exceptionally small children. The lower age limit of the chart is set at 32 weeks' gestation, although it should be used 
Table 3 Correct responses to standard questions for differing severity of growth faltering

\begin{tabular}{|c|c|c|c|}
\hline \multirow[b]{2}{*}{ Question } & \multicolumn{3}{|c|}{$\begin{array}{l}\text { Severity of growth faltering } \\
\text { (chart numbers) }\end{array}$} \\
\hline & $\begin{array}{l}\text { Normal } \\
(1,4,7,10)\end{array}$ & $\begin{array}{l}\text { Moderate } \\
(2,5,8,11)\end{array}$ & $\begin{array}{l}\text { Severe } \\
(3,6,9,12)\end{array}$ \\
\hline \multicolumn{4}{|l|}{ How significant is the fall made by this child? } \\
\hline Not significant & 0 & -1 & -2 \\
\hline Moderately worrying & 1 & 0 & -1 \\
\hline Very worrying & 2 & 1 & 0 \\
\hline \multicolumn{4}{|c|}{$\begin{array}{l}\text { What percentage of children would make a fall down a } \\
\text { centile chart of this size? }\end{array}$} \\
\hline $25 \%$ or more & -1 & -2 & -3 \\
\hline $10 \%$ & 0 & -1 & -2 \\
\hline Less than $5 \%$ & 1 & $\mathbf{0}$ & -1 \\
\hline Less than $1 \%$ & 2 & 1 & $\mathbf{0}$ \\
\hline 1 in 1000 & 3 & 2 & 1 \\
\hline \multicolumn{4}{|c|}{$\begin{array}{l}\text { In the light of today's weight and the previous plotted } \\
\text { weights, what you would plan to do next? }\end{array}$} \\
\hline HV to continue routine monitoring only & $\mathbf{0}$ & -1 & -2 \\
\hline $\begin{array}{l}\text { HV to ask about feeding and/or see again for } \\
\text { reweighing in a month }\end{array}$ & 0 & 0 & -1 \\
\hline \multicolumn{4}{|l|}{ HV to take a detailed dietary/social history and/or } \\
\hline GP to see & 1 & 0 & 0 \\
\hline GP to refer to paediatrician & 2 & 1 & 0 \\
\hline
\end{tabular}

$\mathrm{HV}=$ health visitor

with caution in preterm children, and the upper age limit of the chart was set at 5 years, so that it covers the entire preschool period, as we know that failure to thrive often persists up to that age. ${ }^{10}$ However, since we do not have regression data after the age of 2 years, the later part should not be used to identify new cases after that age, but merely to monitor subsequent progress in children whose problems began before the age of 2 . Such children constitute the great majority of those failing to thrive. ${ }^{9}$

FIELD TESTING

While designing the chart, it was regularly used and discussed in clinical settings to successively refine the layout. An advanced draft was then provided for routine use with existing cases of failure to thrive in Newcastle in the autumn of 1995. One year later, after feedback and some further modifications, the chart was issued to health visitors in Stevenage for routine use in diagnosing and monitoring failure to thrive.

Part 2: evaluating the effectiveness of the new chart

During field testing, a self completion questionnaire was developed to evaluate the impact of the chart on decision making processes. Our

Borderline $\bullet$ Moderate $\Delta$ Severe
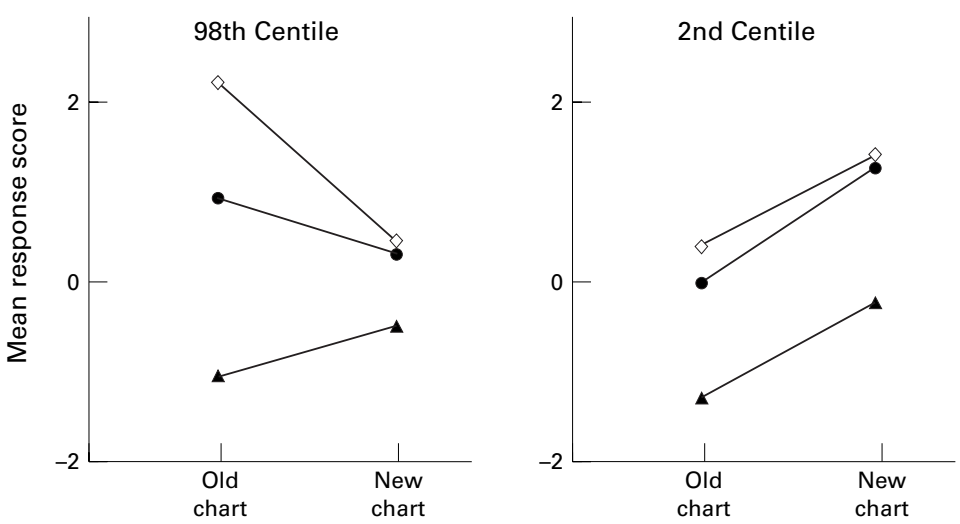

Figure 2 Mean overall response score by size of fall, initial size, and chart type. hypothesis was that the new chart would improve the precision of judgments made, with consequent reduction both in false positives and negatives.

METHODS

Questionnaire

The questionnaire consisted of 12 standardised representations of falls down a centile chart. These varied only in the size of fall, initial size of child, and the chart format (table 2). These were distributed systematically among three versions of the questionnaire, so that each respondent saw four charts, two in the old format and then two in the new format with a page of explanation of the new chart format. No respondents saw the same growth pattern more than once in either format. For each chart, they were asked to answer three standard questions about the significance of the fall made, its prevalence in normal children, and the action that they would propose (table 3 ).

\section{Participants}

Questionnaires were completed during the autumn of 1996 by groups of health visitors in Stevenage, GP principals and registrars in Stevenage and Newcastle before training sessions on failure to thrive, and community paediatricians who were attending a national research meeting. All three versions of the questionnaire were distributed in equal numbers to each group and all questionnaires were handed in before the start of training.

\section{Analysis}

As each respondent rated only four of the 12 charts, the analysis was by chart judgment rather than respondent. For each size of fall, responses were coded for correctness, with 0 constituting a correct answer, negative scores an under reaction, and positive scores an over reaction (table 3). As all three questions were essentially evaluating the respondent's rating of how serious each fall was, the sum of all three was used as an overall response score, with a score of 0 where all three answers were completely correct, but with a wider potential range of \pm 6 .

\section{Results}

Forty four health visitors, 18 GP principals, 10 GP registrars, and 10 community paediatricians completed questionnaires, providing a total of 328 chart ratings. The impact of chart type on judgments was to increase significantly the accuracy of responses to two of the three individual questions and to increase markedly the proportion with completely correct overall scores (table 4). As expected, however, size of fall and initial size influenced responses to the new chart format. Severe falls consistently produced under reaction, which was significantly improved by the new chart. For moderate and borderline falls, large children provoked marked over reaction, which was much improved by the new chart, in contrast with small children who were fairly accurately rated, with the new chart producing over reaction (fig 2). 
Table 4 Correctness of responses to individual questions and overall response score

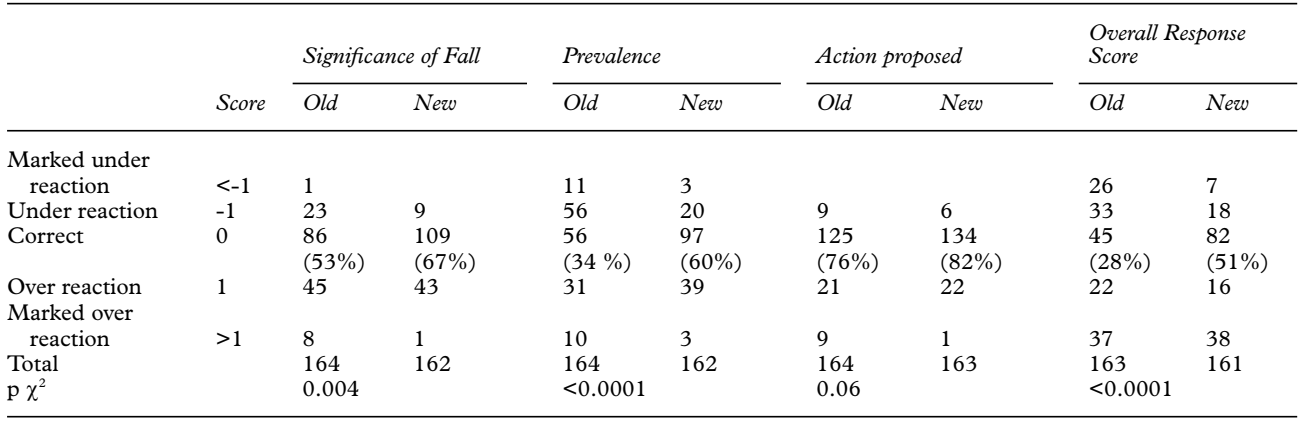

\section{Discussion}

We have described a novel chart which is none the less rooted in the way weight gain is monitored in child health. Field testing of the chart proceeded smoothly, and formal evaluation suggests that it is effective. We do not propose that this chart should replace existing cross sectional charts. It should be used selectively to monitor children whose weight gain appears to be deviating from the expected range to decide whether further investigations are needed and then to observe progress; it can also provide reassurance where falls are in fact within normal limits. The chart is limited by being designed only to monitor weight trends over periods of at least six months from baseline. Some children fall down the chart extremely rapidly and may cross two channel widths in as little as two or three months, in which case the chart can still be used appropriately, but it cannot be used to evaluate lesser variations in weight gain over short time intervals. A problem with short intervals is that the degree of variation between two measurements itself varies with age, with as much variation between birth and 6 weeks of age as there is between 6 weeks and a year. Cole has recently described a new chart to evaluate falls over shorter time intervals, but this has not yet been tested in clinical practice. ${ }^{10}$

Although evaluation of a clinical tool using hypothetical clinical cases can only approximate to its true effectiveness, this approach allows the impact of the chart to be isolated from the influence of severity and initial centile position. We decided not to present the new and old chart formats in random order, although this introduces the possibility of a learning effect, because the explanation and use of the new format would generally increase understanding of what constitutes an abnormal fall. This would modify subsequent responses using the old format.

With these reservations, however, the new chart appears to modify decision making, increasing levels of concern about severe cases of failure to thrive while not leading to over identification of borderline cases. It allows clinicians to diagnose normality with confidence and reduces the possibility of inducing unnecessary parental anxiety. Meanwhile, true cases will be identified, which is useful, as slow weight gain is often responsive to simple dietary advice. It may be a marker of neglect or organic disease, ${ }^{4}$ and predisposes to stunted growth and adult ill health. ${ }^{11}{ }^{12}$

Weight charts have been an established part of paediatric practice for 30 years. With the recent introduction of improved contemporary standards and a more critical approach to child surveillance, it is appropriate to refine the infancy weight chart as a clinical tool. We suggest that the model described here is easy to understand and use and could greatly improve routine practice.

We are grateful to John Matthews and Tim Cole for their important contributions to our thinking and their practical assistance, to the Child Growth Foundation for supplying the cross sectional standards from which the charts are drawn, and to Edmund Hey who advised on successive drafts of the paper. We are particularly grateful to the health visitors of Newcastle and Stevenage for their cooperation.

The weight monitoring chart has now been developed for publication by Harlow Printing Limited, Maxwell Street, South Shields, NE33 4PU.

1 Cole TJ. Conditional reference charts to assess weight gain in British infants. Arch Dis Child 1995;73:8-16.

Hall D. Monitoring children's growth-new charts will help. BMF 1995;311:583-4.

3 Wright CM, Waterston A, Matthews JNS, et al. What is the normal rate of weight gain in infancy? Acta Paediatr 1994; 83:351-6.

4 Wright CM. A population approach to weight monitoring and failure to thrive. In: David TJ, ed. Vol 13. Recent advances in paediatrics. Edinburgh: Churchill Livingstone, 1995:73-87.

5 Emery JL, Waite AJ, Carpenter RG, et al. Apnoea monitors ompared with weighing scales for siblings after cot death. Arch Dis Child 1985;60:55-60.

6 Freeman JV, Cole TJ, Chinn S, et al. Cross sectional stature and weight reference curves for the UK, 1990. Arch Dis Child 1995;73:17-24.

7 Preece M, Freeman J, Cole T. Sex differences in weight in infancy: published centile charts have been updated. $B M \mathcal{F}$ 1996;313:1486.

8 Cole T. Do growth chart centiles need a face lift ? BMf 1994;308:641-2.

9 Wright C. The Parkin Project: a study of screening and intervention in failure to thrive. (MD thesis.) Newcastle upon Tyne, 1996.

10 Cole T. 3-in-1 weight monitoring chart. Lancet 1997;349: $102-3$.

11 Kristiansson B, Fallstrom SP. Growth at age of 4 years subsequent to early failure to thrive. Child Abuse Negl 1987;11: 35-40.

12 Barker D, Winter P, Osmond C, et al. Weight in infancy and death from ischaemic heart disease. Lancet 1989;i:577-80. 